Journal for ImmunoTherapy of Cancer

\section{Intratumor expanded $T$ cell clones can be non-sentinel lymph node derived in breast cancer revealed by single-cell immune profiling}

To cite: Jiao S, Xiong Q, Yan M, et al. Intratumor expanded $T$ cell clones can be non-sentinel lymph node derived in breast cancer revealed by single-cell immune profiling. Journal for ImmunoTherapy of Cancer 2022;10:e003325. doi:10.1136/ jitc-2021-003325

- Additional supplemental material is published online only. To view, please visit the journal online (http://dx.doi.org/10. 1136/jitc-2021-003325).

1

Accepted 23 November 2021

D Check for updates

(C) Author(s) (or their employer(s)) 2022. Re-use permitted under CC BY-NC. No commercial re-use. See rights and permissions. Published by BMJ.

For numbered affiliations see end of article.

\section{Correspondence to}

Dr Tong Liu;

liutong@hrbmu.edu.cn

Dr Da Pang;

pangda@ems.hrbmu.edu.cn

Dr Shiping Jiao;

jiaoshp@tcrximmune.cn

\section{ABSTRACT}

Background Sentinel lymph nodes (LNs) are regarded as key immune surveillance sites in cancer wherein mature dendritic cells present tumor-derived antigens to prime and activate $T$ cells, which then migrate to the tumor site. However, it is unclear whether the tumor-specific $T$ cells can be elicited within the tumor independent of the sentinel LNs.

Methods We performed an integrative analysis of gene expression profiles of 65,285 cells and T cell receptor sequences of $15,831 \mathrm{~T}$ cells from 5 paired primary breast tumors and sentinel LNs to identify where clonal T cells come from and the characteristics of those clonal T cells. Results The proportion of clonal T cells was higher in the primary tumors compared with the sentinel LNs, whereas all expanded clones identified in the sentinel LN were also present in the primary tumors. In contrast, $10.91 \%$ of the expanded clones in the primary tumors were not found in the sentinel LNs. These novel intratumoral T cell clones were characterized by high tissues retention capacity (CXCR6 + ITGAE+) and a distinct coinhibitory pattern (CD39+NKG2A+) compared with the expanded T cell clones common to both sites. Furthermore, multiplex immunofluorescence imaging showed the presence of tertiary lymphoid structures (TLS) in the primary breast tumors wherein the activated cytolytic T cells were concentrated, indicating its possible role in eliciting nonsentinel LN-derived T cell clones.

Conclusions Our study revealed expanded intratumor non-sentinel LN derived T cell clones located in the TLS, which points to the need for exploring the role of TLS in antitumor immunity.

\section{BACKGROUND}

The accumulation of mutations in cancer cells generate tumor-specific antigens that elicit the host immune response. ${ }^{1}$ The antigens shed from the tumor cells are captured by the mature dendritic cells (DCs), which then migrate to secondary lymphoid organs and present the antigenic peptides with the major histocompatibility complex to $\mathrm{T}$ cells and $\mathrm{B}$ cells. Following antigen recognition, the CD4 + T cells promote B cell proliferation in the primary follicles to form a secondary follicle as germinal center, wherein the primed and activated lymphocytes can expand further. The antigen-primed $\mathrm{T}$ cells and B cells eventually egress from the lymph node (LN) and enter the tumor bed. ${ }^{2}$ However, it is unclear whether these tumorspecific $\mathrm{T}$ cell clones are primed only within the sentinel LNs and then migrate to the tumor, or if a proportion of expanded clones can be primed and elicited within the primary tumor sites.

Tertiary lymphoid structures (TLS) in tumors have drawn considerable interest in recent years as a proxy for an immunologically 'hot' environment. Studies show that TLS play a major role in controlling tumor invasion and metastasis, and a high density of TLS correlates with favorable overall survival and disease-free survival in multiple cancer types. ${ }^{3}$ In addition, the presence of B cells and $\mathrm{T}$ follicular helper cells in the TLS correlates with improved prognosis in murine cancer models as well as patients. ${ }^{4-6}$ However, the exact role of TLS, or its potential function as an LN-like structure, in antitumor immunity is unknown.

Single-cell $\mathrm{T}$ cell receptor (scTCR) sequencing allows rapid identification of putative antigen-specific $\mathrm{T}$ cell clones. ${ }^{7} 8$ Furthermore, integrated analysis of TCR and single-cell gene expression data can help track $\mathrm{T}$ cell clones and their transcriptional phenotypes, ${ }^{79}$ and assess the relationship of $\mathrm{T}$ cells in different locations.

Sentinel LN biopsy therapy is concepted and commonly applied in breast cancer surgery. It along with the removal of primary breast tumor is the standard treatment for most, clinically node-negative breast cancer at diagnosis who undergoes conservative surgery as initial treatment. ${ }^{10}$ In addition, 
in the breast cancer mastectomy, the primary breast cancer and the corresponding sentinel $\mathrm{LN}$ are easy to be exposed by the surgeons and yield a clear anatomic relationship particularly with the aid of staining dye. These clinical features provided us the opportunity to collect the primary tumors and the first tumor draining LNs. In this study, we collected primary breast cancer tissues and adjacent sentinel LNs from 5 patients, and performed single cell gene expression profiling and TCR sequencing to determine the relationship between intratumoral and sentinel LN-residing T cell clones.

\section{MATERIALS AND METHODS}

\section{Clinical subjects and clinical sample collection}

Five patients with clinically node-negative breast cancer at diagnosis who undergoes conservative surgery as initial treatment were recruited from Harbin Medical University Cancer Hospital. The demographic characteristics of the study population are listed in online supplemental table S1.

\section{Sentinel LN identification and collection}

A blue dye was injected close to the tumor, and the stained sentinel LNs were detected after $15 \mathrm{~min}$ using a probe. All sentinel LNs (generally 4-6) were collected from each of the 5 patients.

\section{Generation of single cell RNA-seq and V(D)J libraries}

The single cell RNA-seq and V(D)J libraries were generated using the 10X Genomics Chromium Controller Instrument and Chromium single cell 5' library \& gel bead kit, and the V(D)J enrichment kit according to manufacturers' instructions. Briefly, 8000 peripheral blood mononuclear cells (PBMCs) ( $>90 \%$ viability) were loaded on the controller to generate single-cell gel bead-in-emulsions. The mRNA was reverse transcribed and sample indexed to barcoded cDNA, purified using DynaBeads and amplified by PCR. To construct the 5' gene expression library, the amplified barcoded cDNA was fragmented, end repaired, A-tailed, sample indexed and double-sized selected with SPRI beads (average size $450 \mathrm{bp}$ ). For the V(D)J library, human $\mathrm{T}$ cell $\mathrm{V}(\mathrm{D}) \mathrm{J}$ sequences were enriched from the amplified cDNA followed by fragmentation, end repairing, A-tailing, sample indexing and double-sized selection with SPRI beads (average size, $600 \mathrm{bp}$ ). The DNA was quantified, and fragment size distribution of the libraries were determined using the Qubit dsDNA HS assay kit (Thermo, Q32851) and Agilent 2100 BioAnalyzer High Sensitivity DNA kit (Agilent Technologies, 5067-4626). Pooled libraries were then sequenced on an Illumina high output sequencing platform, with $150 \mathrm{bp}$ on the two reads for RNA-seq and V(D)J libraries.

\section{X library sequencing}

The single-cell RNA libraries were sequenced on an Illumina Novaseq to a minimum sequencing depth of 50000 reads per cell using reads lengths of $150 \mathrm{bp}$ read
1, $8 \mathrm{bp} \mathrm{i7}$ index and $150 \mathrm{bp}$ read 2. The scTCR libraries were sequenced on an Illumina Novaseq to a minimum sequencing depth of 10000 reads per cell using reads lengths of $150 \mathrm{bp}$ read $1,8 \mathrm{bp}$ i7 index and $150 \mathrm{bp}$ read 2. For scRNA-seq, 86,401 cells and 7,168,372,158 reads were obtained with an average of $716,837,216$ reads per sample and 82,966 reads per cell, and an average of 19,345 genes were detected per sample. For scTCR-seq, 38,429 cells and 582,432,249 reads were obtained with an average of $58,243,225$ reads per sample and 15,156 reads per cell. The single cell mapping statistics are shown in online supplemental table S2.

\section{Analyses of scRNA-seq data}

The scRNA-seq reads of each sample were independently aligned to the GRCh38 reference genome (10X Genomics, V.3.0.0) and quantified using cellranger count pipeline (10X Genomics, V.3.1.0) with default parameters. Filtered count matrix of features generated from cellranger count were then analyzed using Seurat (V.3.1.5). ${ }^{11}$ Genes expressed in $<3$ cells, cells with $<200$ or $>5000$ expressed genes, or cells with $>10 \%$ mitochondrial counts were discarded. As described by Yost $e t a l^{2}$ batch effects between samples and cells related to $\mathrm{S}$ and G2/M cell cycle phases, heat-shock gene expression signature, number of unique molecular identifiers (UMIs) per cell and mitochondrial RNA content were removed when scaling the data. Variable genes were identified based on average of expression $>0.1$ and dispersion $>1$, and the top 5000 variable genes were analyzed. To avoid clustering based on variable $\mathrm{V}(\mathrm{D}) \mathrm{J}$ transcripts, the variable TCR and immunoglobulin genes were not included. In addition, the S and G2/M cell cycle and heatshock signature genes were also removed to prevent any clustering effect due to both factors. Cells were clustered using a shared nearest neighbor modularity optimizationbased clustering algorithm with the first 50 principal components and resolution set to 0.5 . The clusters were annotated manually based on the differentially expressed genes (DEGs) between clusters (online supplemental tables S3,S4). The differential expression test was performed by FindAllMarkers function in the Seurat package with min. pct $=0.25$, other parameters as default, and only positive markers (only.pos=TRUE). Wilcoxon rank-sSum test was used for statistical analysis.

\section{Analyses of scTCR-seq}

The scTCR-seq reads were independently aligned to the GRCh38 reference genome (10X Genomics, V.3.1.0) for each sample, and the clonotype analysis and TCR annotation were performed using the cellranger VDJ pipeline (10X Genomics, V.3.1.0). The percentage of clonotypes in each sample is calculated as: cells with identical $\mathrm{T}$ cell receptor beta locus (TRB) CDR3/total T cells * 100\%. ${ }^{9}$

\section{Velocity analysis of malignant cells}

RNA velocity is defined as the rate of change of mRNA molecule abundance in the cell. ${ }^{1314}$ The cancer cell clusters $9,12,16,18,19,22$, and 25 with respective cell numbers 


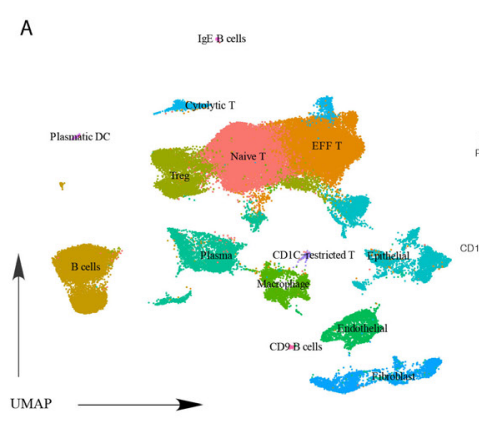

C

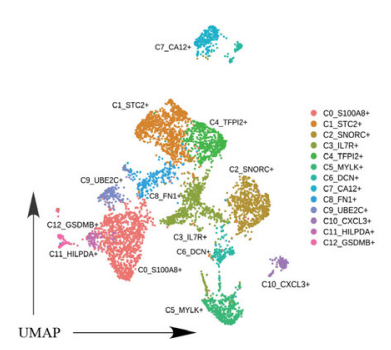

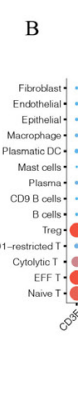

F
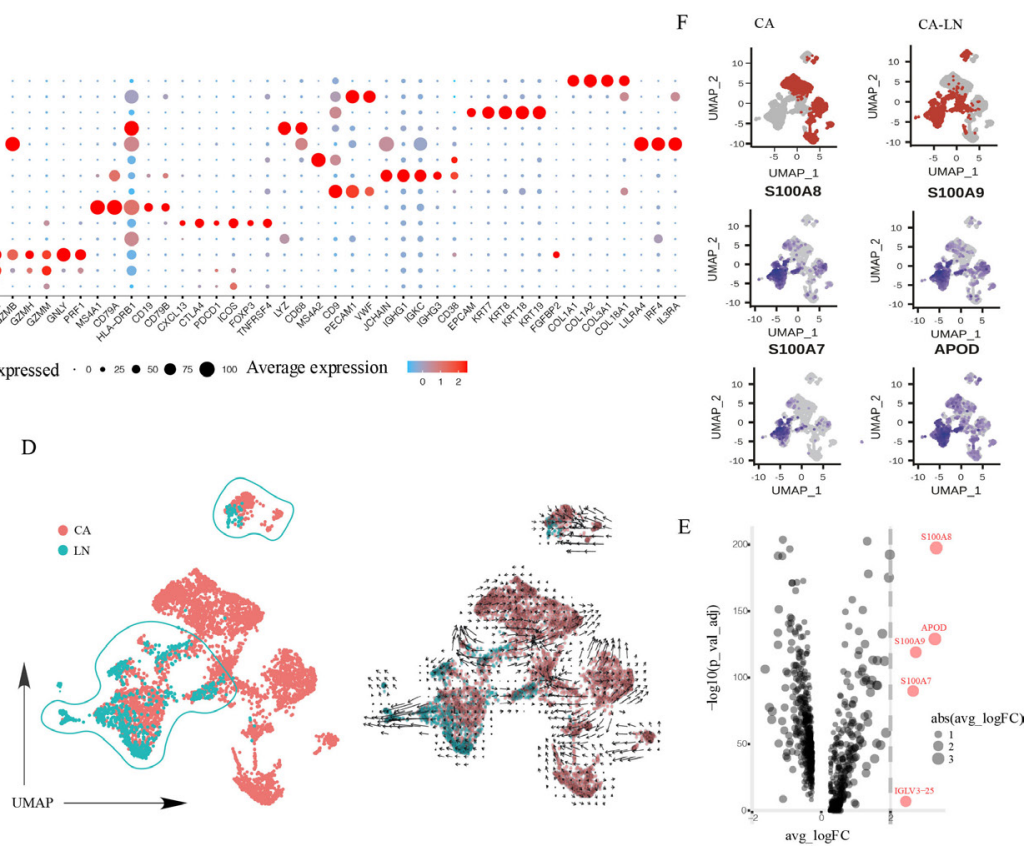

Figure 1 Primary cancer cells inclined to metastasis express high levels of S100A and APOD. (A) UMAP of all cells with each cell type indicated by a color code. (B) Expression of characteristic markers in each cell type. (C) UMAP of malignant cells with color-coded clusters and expression of characteristic genes in each cluster. (D) UMAP of malignant cells from CA (red) and LN (blue), and the velocities (arrows) of malignant cell. (E) Volcano plot of DEGs. Differential expression test was perform by Wilcoxon rank sum test. Significant DEGs with $p<0.05$ and absolute value of avg_logFC $\geq 2$ are indicated in red. (F) Distribution of cancer cells in the pure (CA) and mixed (CA \& LN) areas in the UMAP space and the expression of four DEGs. avg_logFC, log fold-chage of the average expression between the two groups; CA, cancer; DC, dendritic cell; DEGs, differentially expressed genes; LN, lymph node; UMAP, uniform manifold approximation and projection.

1957, 1600, 1035, 445, 300, 140 and 98 were extracted and re-clustered to get higher resolution. Using the optimal resolution of $0.4,13$ clusters were obtained (figure 1) and velocity analysis was performed by velocyto ${ }^{14}$ (python version) with default parameters. The DEGs were identified by FindMarkers function in the Seurat package with default parameters using primary cancer cells with $(\mathrm{C} 0$, C3, C7, C8, C9, C11, C12) and without (C1, C2, C4, C5, C6, C10) migration tendency. The DEGs were visualized by the volcano plot, and the thresholds were $\mathrm{p}<0.05$ and avg_logFC $\geq 2$.

\section{Velocity analysis of $\mathrm{T}$ cells}

The velocity analysis of $\mathrm{T}$ cells was performed using scVelo. ${ }^{13}$ The parameters were set as follows: counts $=20$, n_top_genes $=2000, n \_p c s=30$ and n_neighbors $=30$.

\section{CNV analysis and identification of malignant cells}

Since most breast cancers are carcinomas that arise from the epithelial cells, ${ }^{15}$ the epithelial gene signature was constructed using the top 50 genes with highest correlation to epithelial cell adhesion molecule (EPCAM), and clusters 9, 12, 16, 18, 19, 22 and 25 were annotated by the module scores of these genes. The enrichment of epithelial signature genes in individual cells were scored using the function AddModuleScore in Seurat. The copy number variation (CNV) status of all cells in the epithelial clusters were inferred by R package InferCNV ${ }^{16}$ (https:/ / github.com/broadinstitute/inferCNV) against that of the non-epithelial cells including fibroblasts, endothelial and immune cells. Cluster specific CNVs in malignant cells were visualized by heat maps (online supplemental figure $\mathrm{S} 1 \mathrm{E}, \mathrm{F}$ ), and the malignant and non-malignant cells were distinguished on the basis of their individual CNV score, which was defined as the mean of squared CNV values from hidden Markov model CNV predictions across all genes for each cell. The malignant cells were defined as those with $\mathrm{CNV}$ score greater than the maximum $\mathrm{CNV}$ score of normal cells.

\section{Multiplex immunofluorescence assays}

Multiplex staining was performed using the Opal 4-Color Manual IHC Kit (NEL810001KT) with the anti-CD3 (1:150; ZA-0508, ZSGB-BIO), anti-CD20 (1:100; ZM-0039, ZSGB-BIO), anti-CCL4 (1:50, PA5-23681, ThermoFisher), anti-CD8 (1:100, ab4055, abcam), anti-PNAD (1:100, \#120801, Biolegend), anti-PD1(1:100, ab52587, Abcam) and anti-CD103 (1:100, ab254017, Abcam) antibodies with subsequent visualization with fluorescein AF-690 (1:75), AF-520 (1:75) and AF-570 (1:50). The nuclei were counterstained with 4', 6-diamidino-2-phenylindole $(1: 3,000)$. All sections were covered with Vectashield Hardset 895 mounting media, and scanned using the Vectra slide scanner (PerkinElmer).

\section{TCR T construction}

$\mathrm{T}$ cells were isolated from peripheral blood mononuclear cells from the patient by CD3/CD28 bead pulldown and 
incubated overnight with $\mathrm{CD} 3 / \mathrm{CD} 28$ antibody-coated beads in the presence of 100 units/mL rh interleukin-2. Activated T cells were lentivirally transduced to express the patient-derived TCR sequences or vector control, as previously described. ${ }^{8}$ The VDJ sequences of transduced TCR sequence were derived from the patient's four expanded T cell clones or four clones represented by only one cell. The mouse TCR alpha and beta constant domains were utilized to construct the full length of TCR to decrease the possibility of mispairing with endogenous TCR alpha or beta chains. The transduced $\mathrm{T}$ cells expanded for 5 days.

\section{INTERFERON $\Gamma$ ELISA}

The 96-well plate was coated with interferon $\gamma$ (IFN $\gamma$ ) capture antibody for 48 hours. A total of $1 \times 10^{5}$ TCR-T cells per well cultured with $2 \times 10^{5}$ autologous tumor cells $(1 \times 105)$ for 24 hours in the plate. Supernatants were collected and analyzed for IFN $\gamma$ using the Human IFN $\gamma$ DuoSet ELISA (R\&D Systems), with the use of a luminescent HRP substrate (Glo Substrate, R\&D Systems). The signal was measured using the FLUOstar Omega plate reader (BMG Labtech).

\section{RESULTS}

\section{Cell-type composition in primary breast cancers and paired} sentinel LN

Single cell suspensions of primary breast tumors and paired sentinel LNs of 5 patients (P1-P5) were prepared (figure 2A and online supplemental table S1). All patients had histologically confirmed tumors, and metastatic cancer cells were detected in at least one sentinel LN of each patient (figure 2B and online supplemental table S1). Single cell gene expression analysis and TCR sequencing were performed using the $10 \mathrm{X}$ genomics platform. After quality control (online supplemental figure S1A,B), 32,923 cells from the tumor tissues and 32,362 cells from the corresponding sentinel LNs were clustered into 27 clusters (online supplemental figure S1C) and annotated as 14 subsets: Naive T cells (CD3E+, IL7R+, CCR7+), effector (EFF) T cells (CD3E+, CCL5+, NKG7+, GZMK+, GZMA+), Cytolytic T cells (FGFBP2+, NKG7+, GZMA+, GZMB+, GZMM+, GNLY+, PRF1+, CCL5+), CD1-restricted T cells (CD1E+, CCR7+, IL7R+), Treg cells (CD4+, FOXP3+, TNFRSF4+, CTLA4+), B cells (CD19+, MS4A1+, HLA-DRB1+), CD9 B cells (CD9+, PECAM1+), Plasma cells (JCHAIN+, IGHG1+, IGKC+, CD38+, CD19-), Mast cells (MS4A2+), Plasmatic DCs (LILRA4+, IRF4+, IL3RA+), Macrophage (CD68+, HLADRB1+, LYZ+), Epithelial cells (EPCAM+, KRT7+, KRT8+, KRT18+, KRT19+), Endothelial cells (PECAM1+, VWF+), and Fibroblasts (COL1A1+, COL1A2+, COL3A1+, COL18A1+) (figure 1A,B). All 14 subsets were identified in breast tumors and the sentinel LNs. The proportion of antigen-experienced $\mathrm{T}$ cells (EFF $\mathrm{T}$ cells and cytolytic $\mathrm{T}$ cells) were higher in tumors, whereas B cells were more abundant in the LNs (online supplemental figure $\mathrm{S} 1 \mathrm{C}, \mathrm{D})$. This is consistent with the large number of B cells

A

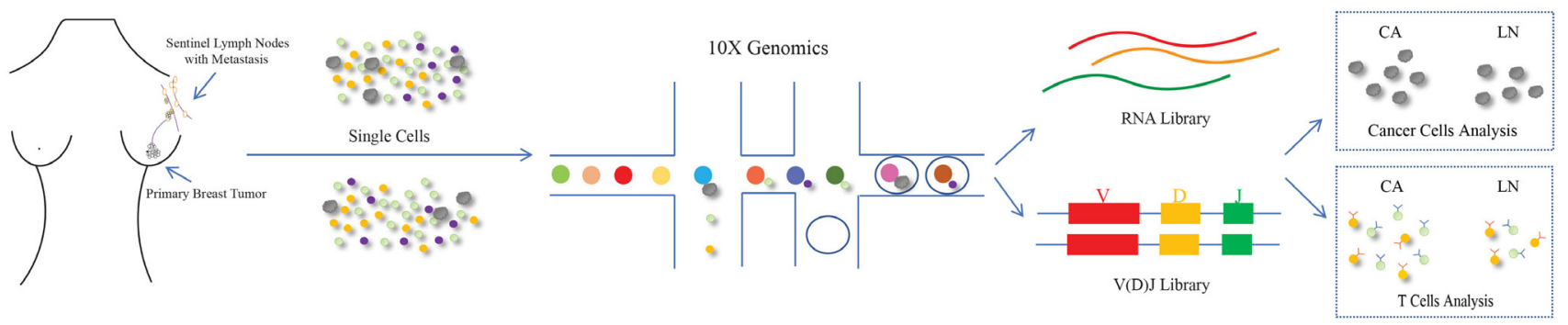

B

CAl

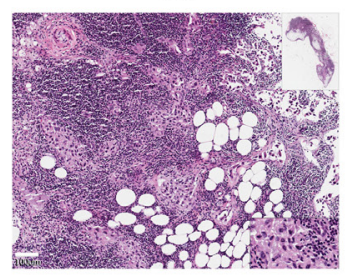

LN1

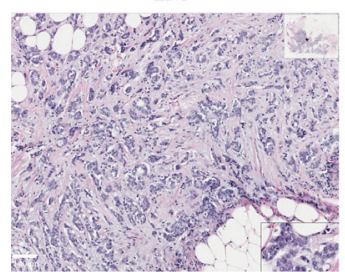

$\mathrm{CA} 2$

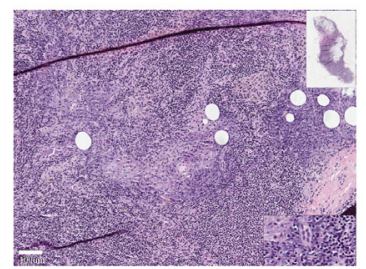

LN2

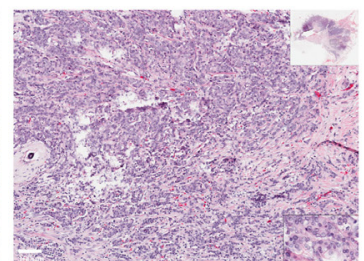

CA3

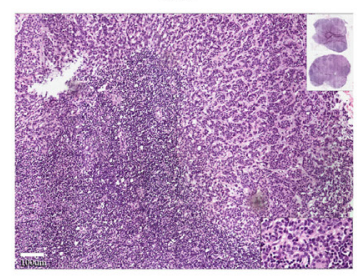

LN3

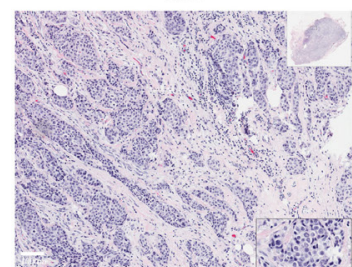

CA4

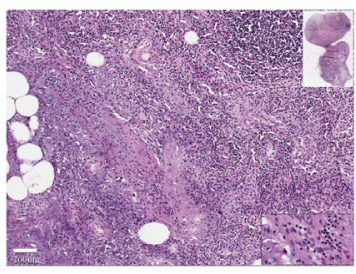

LN4

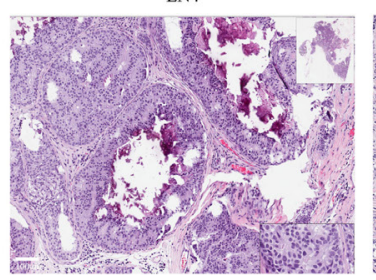

illustration of the analyses performed in this study. (B) Representative images of HE-stained primary breast tumors and sentinel LNs of 5 patients. LN, lymph node; TCR, T cell receptor. 
observed in the germinal centers of LNs, and the enrichment of antigen-experienced $\mathrm{T}$ cells in the tumor sites.

\section{Primary breast cancer cells inclined to metastasis express high levels of S100A and APOD}

Studies show that CNV patterns can be used to distinguish between the malignant and non-malignant cells. ${ }^{917}{ }^{18} \mathrm{We}$ calculated a CNV score for each cell and used this score to distinguish the malignant cells from non-malignant cells. We found that all 27 clusters (online supplemental figure S1C) contained exclusively malignant or non-malignant cells, and the epithelial clusters are pure malignant cells. This is consistent with another study's finding that epithelial and CNV-based classifications were highly concordant and cross-validated. ${ }^{17}$

The identified malignant cells from tumor tissues and sentinel LNs were reclustered into 13 subsets (figure 1C). The clusters C1, C2, C4, C5, C6 and C10 were mainly composed of cancer cells from primary tumor sites (CA area), while clusters C0, C3, C7, C8, C9, C11 and C12 consisted of cancer cells from both CA \& LN areas (figure 1D and online supplemental figure S1G). The proximity between the mixed CA \& LN primary cancer cells and metastatic cancer cells on the uniform manifold approximation and projection (UMAP) indicated their high transcriptomic similarity. RNA velocity analysis showed a trend that cancer cells in primary tumors concentratedly pointed to those in LNs (figure 1D), indicating that these cancer cells would likely develop to the status of those in the sentinel LNs. In contrast, the velocity directions in the pure primary cancer cell populations were diverse. This indicates that the primary cancer cells in the mixed area are more inclined to metastasize to the LNs. Finally, differential gene expression analysis between metastasis-inclined cancer cells and those of CA areas showed that S100A7, 8 and 9 and APOD were significantly upregulated in the metastasis-inclined cancer cells, with S100A8 (avg_logFC=3.32, p_val_adj=3.02E-198) exhibiting the maximum increase in expression levels (figure 1E,F). It has been documented that S100A7, S100A8, and S100A9 are key molecules in cell motility and promote the migration and metastasis of multiple cancers. ${ }^{1920}$ APOD has been found to confer an increased risk of developing metastatic breast cancers. ${ }^{20}$

\section{Characteristics of $\mathrm{T}$ cells residing in primary breast cancers and paired sentinel LNs}

We further profiled the $T$ cells residing within the tumors and in paired sentinel LNs with metastasis. A total of 15831 T cells with both transcriptome and TCR information from tumor tissues (8665 cells) and paired sentinel LNs (7166 cells) of 5 breast cancer patients were batcheffect corrected (online supplemental figure S2A,B) and clustered into 11 clusters (figure 3A). Clusters 4, 1, 3 and 5 were annotated as Naive T cells (LEF1+, CCR7+, TCF7+), Naïve EFF T cells (CCR7+, TCF7+, CD69+), Treg cells (CD4+, FOXP3+, CD25+, ICOS+, CTLA4+) and $\mathrm{T}$ follicular cells (CD4+, TOX+, CXCL13+, PDCD1+,
ICOS+) (figure 3C, online supplemental table S3). Naïve $\mathrm{T}$ cells and Naïve EFF T cells mainly comprised of CD4 $\mathrm{T}$ cells along with a small proportion of CD8 $\mathrm{T}$ cells. Cluster 10 was characterized by high expression levels of the cytotoxic granules granzyme B (GZMB), granzyme A (GZMA), granzyme H (GZMH), perforin 1 (PRF1) and granulysin (GNLY), and very low levels of inhibitory molecules. Cluster 10 was annotated as Cytolytic T cells with strong cytotoxic capacity. Cluster 9 also expressed high levels of cytotoxic granules but had lower GZMA, PRF1 and GNLY expression compared with cluster 10. In addition, cluster 9 also expressed the co-inhibitory molecules programmed cell death 1 (PDCD1), hepatitis A virus cellular receptor 2 (HAVCR2), T cell immunoreceptor with Ig and ITIM domains (TIGIT), cytotoxic T-lymphocyte associated protein 4 (CTLA4) and lymphocyte activating 3 (LAG3), which was indicative of a dysfunctional or inclined to dysfunctional status. Therefore, we annotated this cluster as dysfunctional $\mathrm{T}$ cells.

The majority of CD8 T cells (clusters $0,2,6,7$ and 8) were antigen experienced, as indicated by the presence of CD69+, CCL5 +and NKG7+, and mainly expressed GZMK. In contrast, the GZMB, GZMH, PRF1 and GNLY levels in these antigen-experienced $\mathrm{T}$ cells were significantly lower compared with those in Cytolytic T cells and Dysfunctional $\mathrm{T}$ cells. To further explore the plasticity of antigenexperienced T cells, we performed a velocity analysis. ${ }^{13} 14$ RNA velocities (arrows) of all $\mathrm{T}$ cells were projected and visualized in the UMAP space (figure 3B). Cells in cluster 8 flowed to either cluster 9 (dysfunctional $\mathrm{T}$ cells) or cluster 10 (cytolytic $\mathrm{T}$ cells). The antigen-experienced $\mathrm{T}$ cells (clusters $0,2,6,7,8$ ) contained several 'velocity centers' that pointed to different cell states like cytolytic, dysfunctional or naive, indicating a high plasticity of this population. Therefore, we annotated this population as plastic transitional EFF T cells.

The CD8 $+\mathrm{T}$ cells were then plotted on the diffusion map and the clustering information of UMAP was integrated. The Cytolytic T cells, Dysfunction T cells and Naïve $T$ cells were consistently aggregated into three separate branches (figure 3D), indicating their divergent status. The plastic Transitional EFF T cells (clusters $0,2,6,7$ and 8) were aggregated in the intersection of the three branches. To further identify potential markers of the functional status of the intratumoral and sentinel LN T cells, we analyzed the average expression levels of naive, cytolytic and dysfunctional status-related makers in cells along the first or second component of the diffusion map (figure 3E). All candidate markers were selected from the list of DEGs (online supplemental table S4). As shown in figure $3 \mathrm{~F}$, fibroblast growth factor binding protein 2 (FGFBP2), HAVCR2 and lymphoid enhancer binding factor 1 (LEF1) were specific to the cytolytic, dysfunctional, and naïve populations. The activation and exhaustion scores of the cells were next calculated based on the respective signature genes that showed correlation to FGFBP2 (activation) and HAVCR2 (exhaustion). We plotted the expression of FGFBP2, HAVCR2 and LEF1 


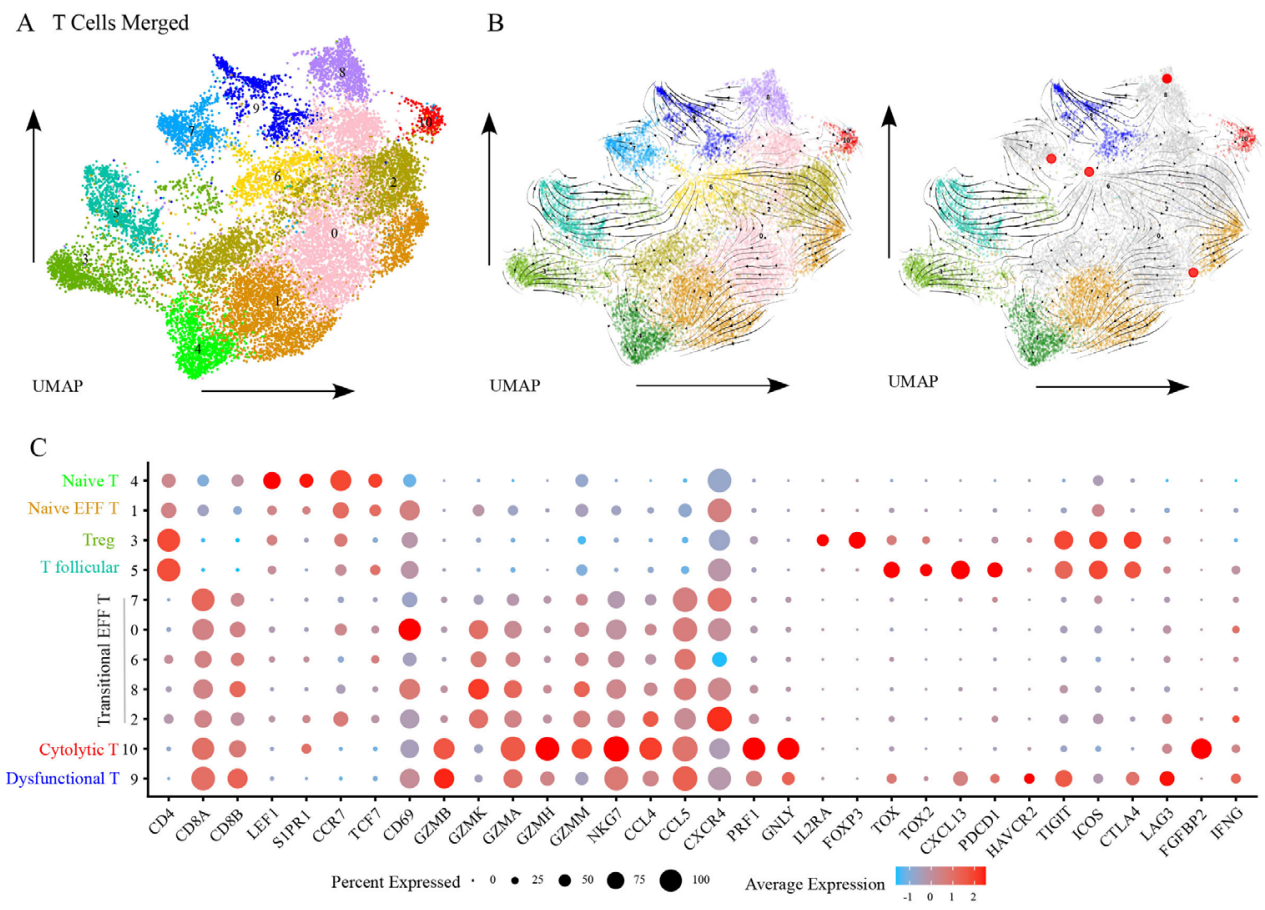

Figure 3 Characteristics of T cells residing in primary breast cancers and paired sentinel LNs. (A) UMAP of T cells with color-coded clusters. (B) Velocities (arrows) of T cells in the different clusters. (C) Expression of characteristics genes in each T cell subset. (D) Diffusion map of all CD8 +T cells. (E) Average expression of naïve, cytolytic and exhaustion markers across diffusion component one and diffusion component 2. (F) Diffusion map of cytolytic (FGFBP2), exhaustion (HAVCR2) and naïve (LEF1) markers. (G) Scatter plots of T cells in the space of activation and exhaustion scores color-coded by expression levels of FGFBP2, HAVCR2 and LEF1. DC, dendritic cell; FGFBP2, fibroblast growth factor binding protein 2; HAVCR2, hepatitis A virus cellular receptor 2; LEF1, lymphoid enhancer binding factor 1; LN, lymph node; UMAP, uniform manifold approximation and projection. 
into the space of $\mathrm{T}$ cell activation-exhaustion, and found that they represented the cytolytic, dysfunctional, and naive status of cells (figure 3G).

\section{Cytolytic T cell population showed the strongest expansion, migration and transition capacity}

Integrated analysis of the single-cell gene expression levels and TCR seq of intratumoral and sentinel LN T cells showed clonal expansion of multiple TCR clonotypes located in the antigen-experienced $\mathrm{T}$ cell area in the UMAP plot (figure 4A,B). The clonal expansion was defined as that the proportion of $\mathrm{T}$ cells with identical TRB CDR3 sequences in total $\mathrm{T}$ cells of the sample is larger than $1 \%$, which are also most likely to be tumorreactive $\mathrm{T}$ cells. ${ }^{79}$ All the identified clonally expanded $\mathrm{T}$ cells were $\mathrm{CD} 8+\mathrm{T}$ cells, and the intratumoral $\mathrm{T}$ cells showed greater clonality compared with those in sentinel LNs (figure 4B). When compared the clonotype sharing between primary tumor sites and corresponding sentinel $\mathrm{LN}$, we found a large number of overlapping clonotypes in antigen-experienced T cells, Treg and T follicular cells population of these two sites, while in Naïve and Naïve EFF $\mathrm{T}$ cell populations, the clonotype sharing is much less (figure 4C,E). In addition, we found that most of expanded clones were common to the primary tumors and sentinel LNs, and all expanded clones in the latter were also identified within the tumor (figure 4D), which is consistent with the hypothesis that antitumor T cells are primed in the sentinel LNs before migration to primary tumors.

STARTRAC was performed to check the degree of clonal expansion, tissue migration and transition states of the T cell subsets in terms of the expa, migr and tran indices respectively. ${ }^{21}$ We found that the cytolytic $\mathrm{T}$ cell population showed the most significant expansion, migration and transition ability (figure $4 \mathrm{~F}$ ), indicating that these cells may constitute the most active antitumor $\mathrm{T}$ cell population.

\section{Intratumoral dominant cancer-specific $\mathrm{T}$ cell clones are potentially derived from non-sentinel LNs}

Based on the distribution and frequencies of the clonally expanded $\mathrm{T}$ cell populations in tumor tissues and sentinel LNs, we classified the TRB clones into the novel, expanded, persistent and contracted types. Novel clones were only detected in primary tumors, whereas the expanded or contracted clones referred to their significant expansion and contraction in the primary tumor sites $(p<0.05)$. The persistent clones had a similar abundance in both tumors and sentinel LNs, and at least one cell was detected at each site. All expanded clones in the five sentinel LN samples were also identified within their paired tumors (figure 4D). In order to show the tumor reactivity of the expanded $\mathrm{T}$ cell clones, we constructed eight TCR T cells of one patient, with four TCR sequences from the expanded $\mathrm{T}$ cell clones and another four from the clonetypes represented by only one cell (online supplemental figure S3A). We found all of the 4 TCRs of expanded T cell clones can recognize autologous tumor cells of the patient, and the tumor reactivity was not observed in the other four clonetypes as control (online supplemental figure S3B). These findings further support the hypothesis that the antigen-loaded DCs or metastatic cancer cells present the tumor antigens to prime $\mathrm{T}$ cells in sentinel LNs, which then migrate to the peripheral tumor site. Some expanded clones were identified as novel clones in tumors (figure 5A), indicating that the intratumoral environment can also prime and activate tumor-specific $\mathrm{T}$ cell clones.

\section{The novel $\mathrm{T}$ cell clones in breast tumors express the coinhibitory CD39 and NKG2A}

These $\mathrm{T}$ cell clones were then projected on the diffusion map, which revealed that a significant proportion of novel clones $(32 \%)$ were located on the highly dysfunctional branch, followed by the contracted (12\%), persistent $(6 \%)$ and expanded clones (3\%). Most of expanded clones $(88 \%)$ resided in the transitional state (figure 5B,C).

The novel $\mathrm{T}$ cell clones were further characterized by differential gene expression analysis against the expanded, persistent and contracted clones. killer cell lectin like receptor C1 (KLRC1/NKG2A), CTLA4, C-X-C motif chemokine receptor 6 (CXCR6), ectonucleoside triphosphate diphosphohydrolase 1 (ENTPD1/CD39) and integrin subunit alpha $\mathrm{E}$ (ITGAE) were significantly upregulated, whereas interleukin 7 receptor (IL7R) and granzyme K (GZMK) were downregulated in the novel clones compared with the other three (figure 5D). CXCR6 and ITGAE mediate tissue retention and residence of $\mathrm{T}$ cells, and their high expression levels indicate that the novel clones are likely elicited in the tumor rather than the sentinel LNs. ENTPD1 (CD39) and KLRC1 (NKG2A) are inhibitory receptors expressed on the activated $\mathrm{T}$ cells $^{22}{ }^{23}$ along with CTLA4. ${ }^{24}$ Their high expression level in the novel clones suggests that the intratumorally elicited clones may be more dysfunctional and inhibitory compared with those in sentinel LNs. In addition, the downregulation of IL7R and GZMK genes indicates that novel clones are less likely to be long-term surviving and plastic. ${ }^{25} 26$

\section{The TLS in primary breast cancer may induce intratumoral novel $\mathrm{T}$ cell clones}

We hypothesized that the novel clonal $\mathrm{T}$ cells may be primed in the intratumoral lymphoid-like structures. To this end, we first analyzed differential gene expressions of expanded clonal CD8 T cells vs antigen experienced non-clonal CD8 T cells and identified C-C motif chemokine ligand 4 (CCL4) as the feature gene of the former (figure 6A). We next performed multiplex immunofluorescence imaging to locate CCL4 +CD8+T cells, which represent clonal $\mathrm{T}$ cells, in the primary tumors (figure 6B). We also included CD20 markers in the multiplex immunofluorescence panel to facilitate us to identify TLS. We found that CCL4 +CD8+T cells are highly 
A

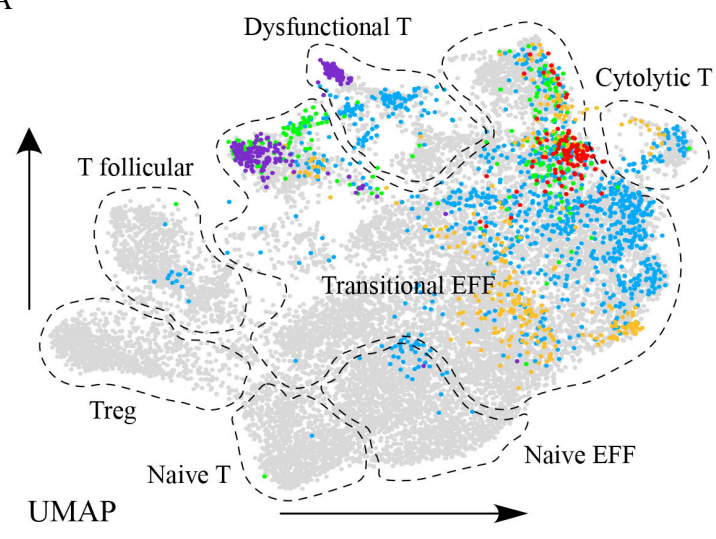

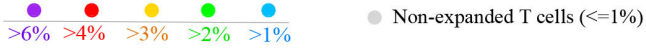

$\mathrm{C}$

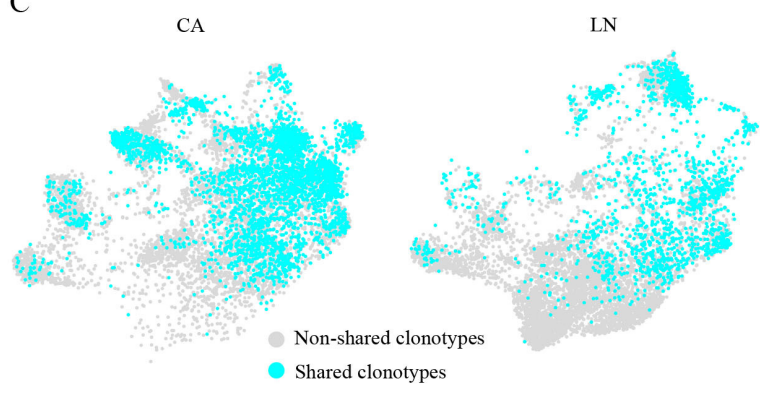

$\mathrm{D}$

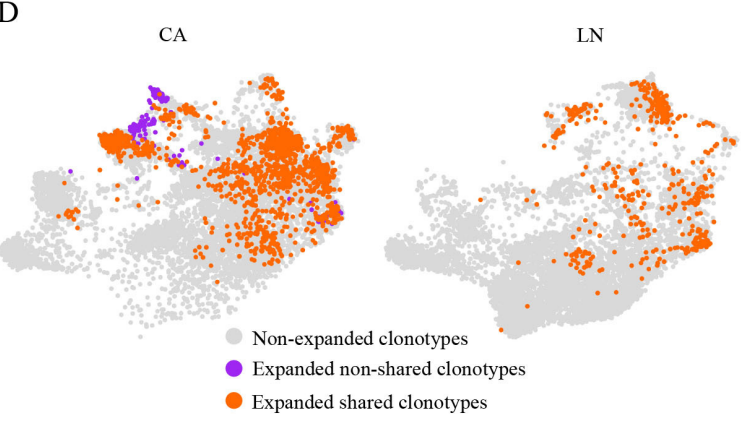

B
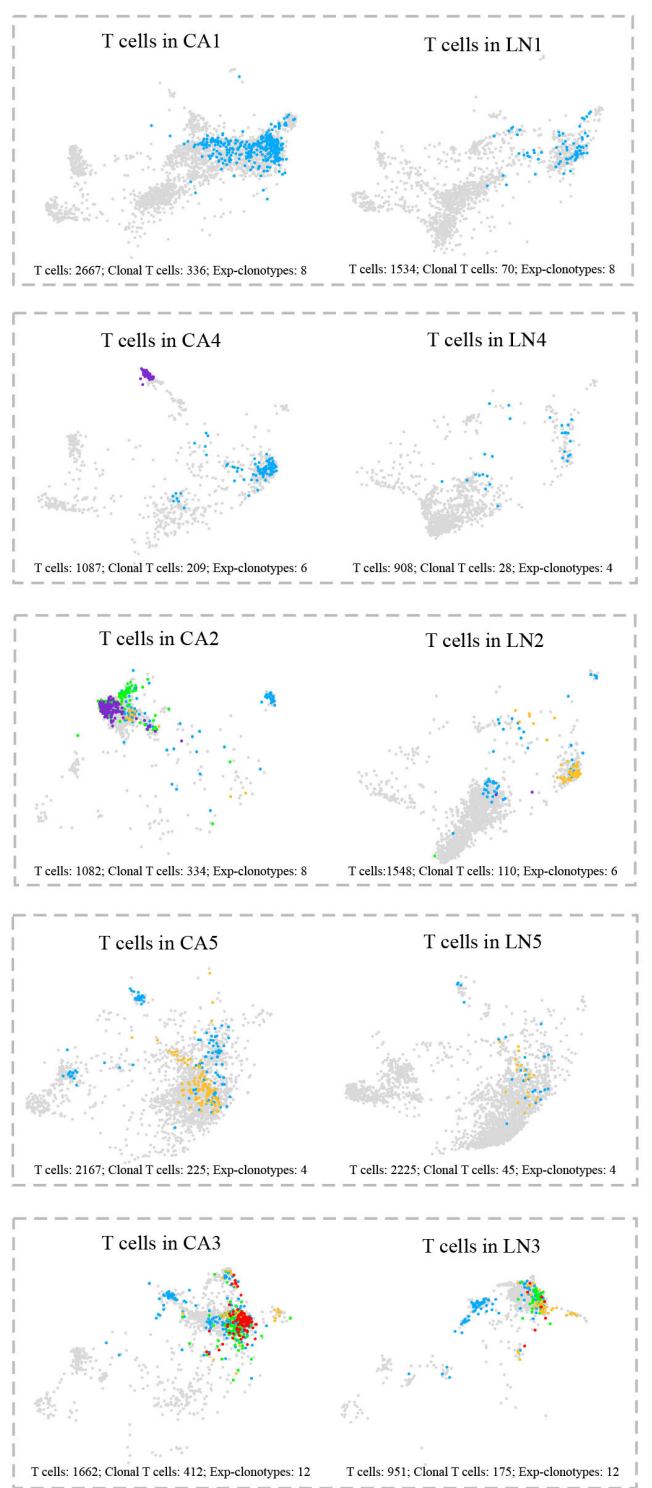

$\mathrm{F}$
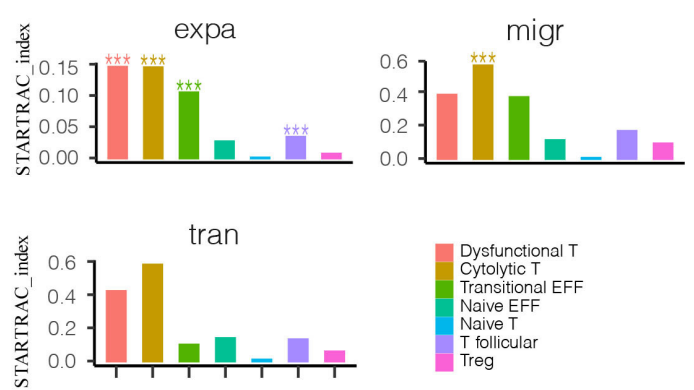

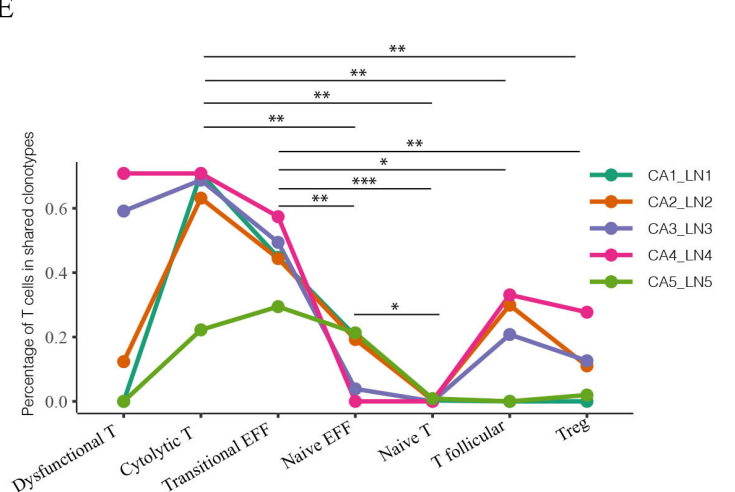

Figure 4 Cytolytic T cell population showed the strongest expansion, migration and transition capacity. (A) UMAP of T cells color-coded by percentage of TRB clonotypes. (B) UMAP of T cells from primary tumor and paired sentinel LN in each patient, color-coded by percentage of TRB clonotypes. Exp-clonotype: expanded clonotype. (C) UMAP of T cells in shared and non-shared TRB clonotypes between primary tumors and sentinel LN. (D) UMAP of T cells in shared expanded, non-shared expanded clonotypes between primary tumors and sentinel LN, and non-expanded clonotypes. (E) Percentage of T cells in shared clonotypes between primary tumors and sentinel LN for each patient in each T cell subset, significance was measured by t-test after natural log-transformed percentage values. ${ }^{\star} \mathrm{P}<0.05$, ${ }^{\star \star} \mathrm{p}<0.01,{ }^{* \star \star} \mathrm{p}<0.001$, respectively. (E) STARTRAC expa, migr, and tran indices of each T cell subset, ${ }^{\star \star \star} \mathrm{p}<0.001$. CA, cancer; $\mathrm{LN}$, lymph node; TRB, T cell receptor beta locus; UMAP, uniform manifold approximation and projection. 


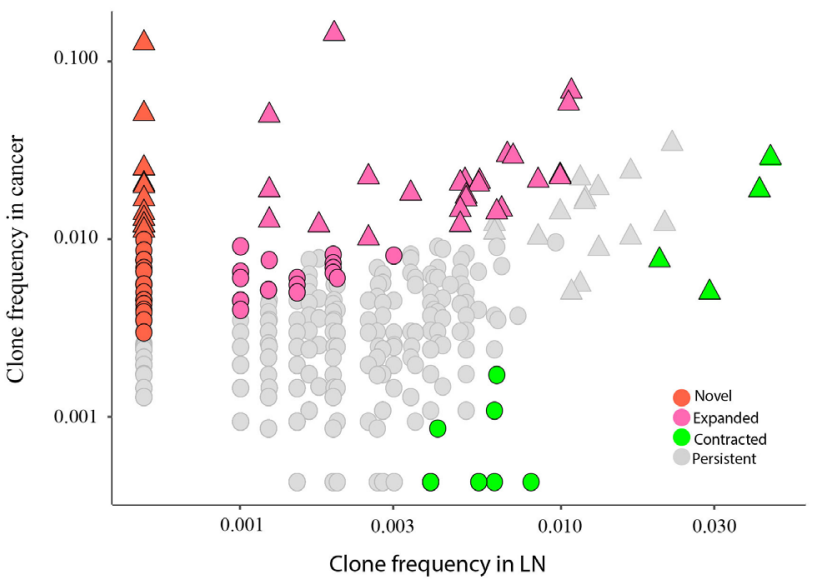

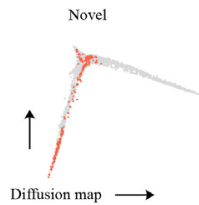
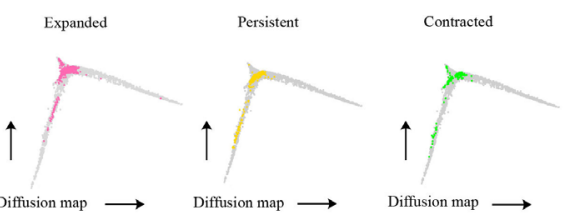

C
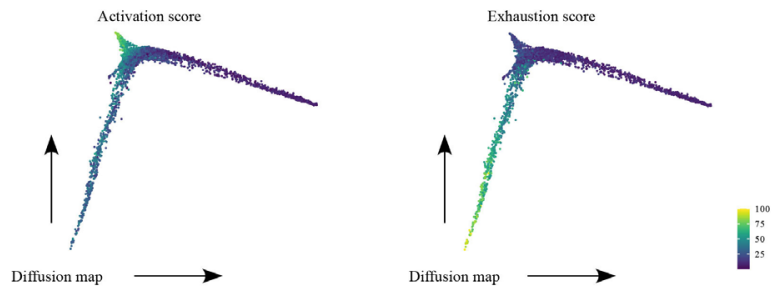

Novel vs Contracted

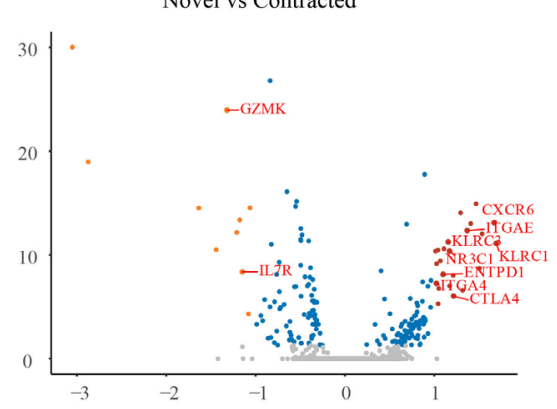

D

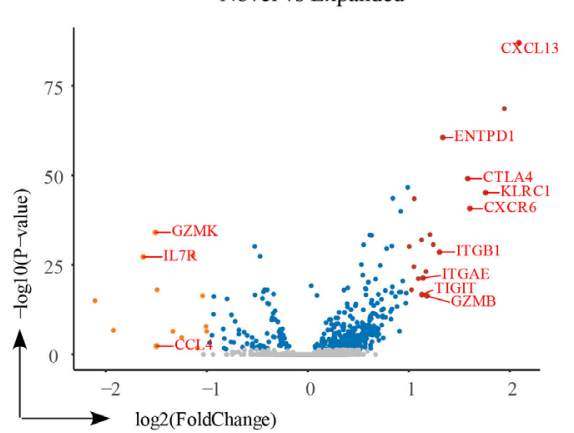

Novel vs Persistent

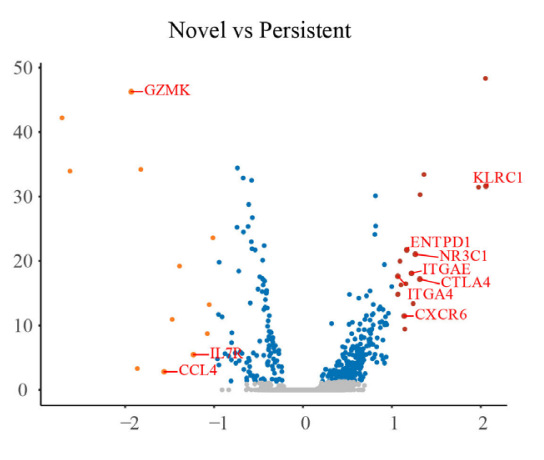

Figure 5 Intratumoral dominant cancer-specific T cell clones are potentially derived from non-sentinel LNs. (A) Frequencies of novel, expanded, persistent, and contracted clones; clones with TRB frequency $>0.01$ in tumors or sentinel LN are marked as triangle, others as circle. (B) Diffusion map of novel, expanded, persistent and contracted clones. (C) Diffusion map of activation and exhaustion scores. (D) Differentially expressed genes between novel and expanded, persistent and contracted clones. Significant DEGs were screened by $p<0.05$ and avg_FC $>=2$. DEGs, differentially expressed genes; LN, lymph nodes; TRB, T cell receptor beta locus.

enriched and aggregated in the TLS, indicating the potential role of TLS in priming non-sentinel LN derived clones. To collaborate the hypothesis, we co-stained PNAD (high endothelial venules marker), CD3, and CD20 and found the existence of HEV in TLS indicating the possibility of naive $\mathrm{T}$ cell infiltrating tumors from the circulation (online supplemental figure S4A). In addition, we performed costaining of CD103 (DC marker), $\mathrm{CD} 8$, and PD1 in the primary breast tumor to show the colocalization of antigen presenting cells and activated $\mathrm{T}$ cells (online supplemental figure $\mathrm{S} 4 \mathrm{~B}$ ). The evidence supported the possibility that tumor reactive $\mathrm{T}$ cells can be primed in TLS of primary tumors.

\section{DISCUSSION}

Conventionally, the tumor-specific $\mathrm{T}$ cell clones are primed in the sentinel LNs following antigen presentation by the mature DCs, and migrate thereafter to the tumor. ${ }^{2}$ In this study, we found that $10.91 \%$ of the expanded $\mathrm{T}$ cell clones in primary tumors were not present in the sentinel LNs, whereas all expanded $\mathrm{T}$ cell clones in the latter were shared with the tumor site. This suggests that novel intratumoral $\mathrm{T}$ cell clones are antigen-primed and elicited within the tumor. However, we cannot exclude the possibility that these clones may be primed in distant LNs and migrate to tumor bed, despite little evidence supporting this hypothesis. Another possibility is that these clones might be primed in bone marrow and move to tumor. Several studies have indicated that tumor antigens can be released into blood and circulate to the bone marrow, where resident DCs can process and present tumor antigens to $\mathrm{T}$ cell and prime them. ${ }^{27}$

The novel $\mathrm{T}$ cell clones in the tumors are characterized by high tissue retention capacity (CXCR6 +ITGAE + ) and dysfunctional tendencies (CD39 +NKG2A+CTLA4+) compared with the clones common to both primary tumors and sentinel LNs. The high tissue retention is in line with the hypothesis that the novel clones are educated and elicited in the primary tumor. CD39 and NKG2A are newly identified immune checkpoint molecules. CD39 inhibits $\mathrm{T}$ cell function in the tumors by converting ATP to the immunomodulatory adenosine. ${ }^{22}$ NKG2A is coexpressed with the tissue-resident molecule CD103 on the $\mathrm{T}$ cells, and inhibits their function on binding with the HLA-E receptor. ${ }^{23}$ The high expression levels of both receptors indicated that the novel $\mathrm{T}$ cell clones elicited in the tumor have divergent coinhibitory patterns compared with the conventional anticancer $\mathrm{T}$ 
A

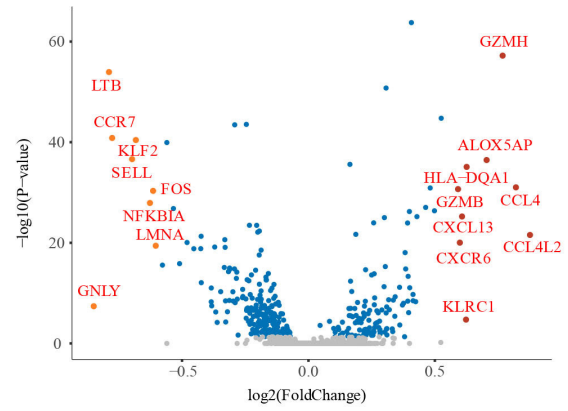

CA3

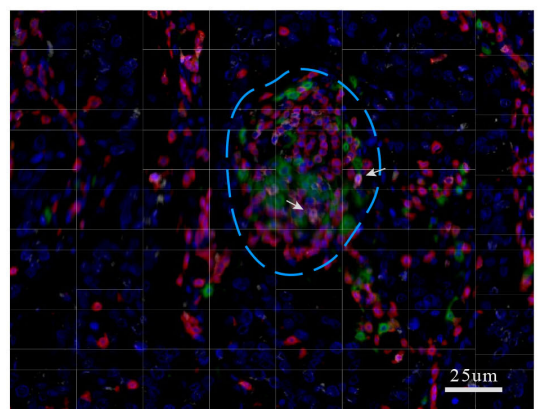

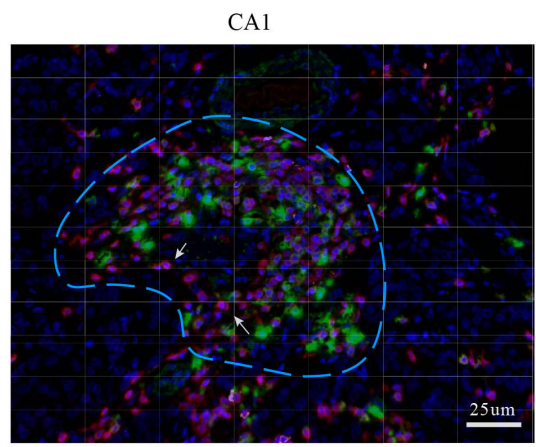

CA4

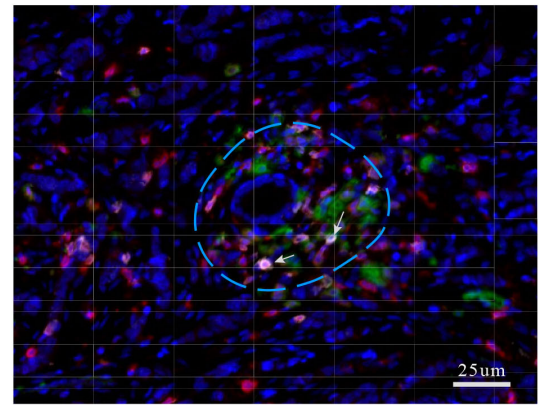

CA2

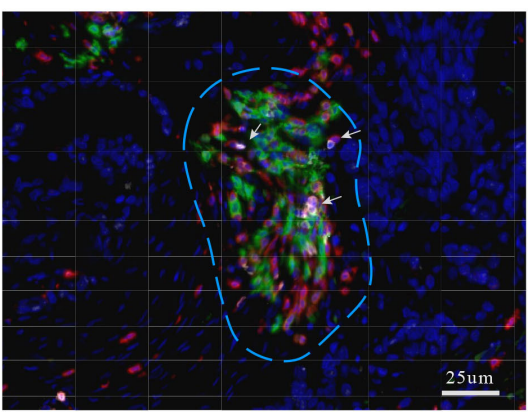

CA5

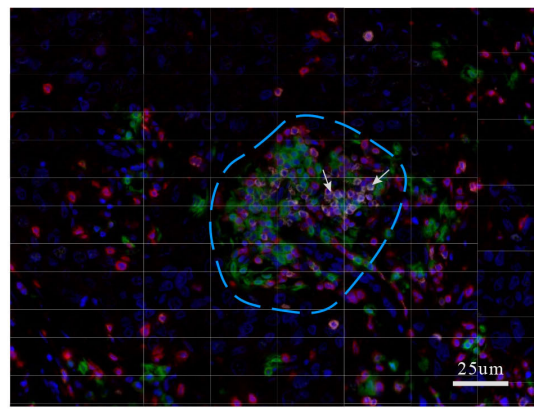

Figure 6 The TLS in primary breast cancer may induce intratumoral novel T cell clones. (A) DEGs between expanded clonal CD8 T cells and antigen-experienced non-clonal CD8 T cells. Significant DEGs were screened by $p<0.05$ and avg_FC $>=1.5$. (B) Multiplex immunofluorescence imaging of tumor tissues. Red - CD3+, white - CCL4+. CD3 +CCL4+ is the signature of clonal effector T cells. Green - CD20 +B cells. The aggregation of T and B cells represents TLS. CA, cancer; DEGs, differentially expressed genes; TLS, tertiary lymphoid structures.

cells that migrate from the LNs, providing a new strategy to reinvigorate anticancer immunity.

The novel T cell clones within tumor can be non-sentinel LN derived T cells, which suggests that sentinel LN-like structures may exist within the tumor that can prime, activate and expand naive $T$ cells. The tumor microenvironment receives immune cells from the peripheral blood as well as the LNs.

When the blood circulation carries plenty of naive $T$ cells through the tumor environment, the ones with suitable binding affinity with tumor antigen can be activated, primed and reside within sentinel LN like structure.

The naive $\mathrm{T}$ cells introduced by the circulating blood includes the ones with suitable binding affinity with tumor antigen, which are then retained, primed and activated within these sentinel LN like structures. Recent studies have shown that the presence of TLS in tumors increases the efficacy of cancer immunotherapy. ${ }^{528}$ Based on our findings, we hypothesize that the TLS function as sentinel LN-like structures that elicit an antitumor $\mathrm{T}$ cell response. The successful priming of antitumor T cells largely depends on the function of DCs. ${ }^{29}{ }^{30}$ Although TLS in the tumors can potentially prime $\mathrm{T}$ cells, the role of DC maturation in this context remains to be elucidated. Future studies should focus on the factors that are essential for priming tumor-specific $\mathrm{T}$ cells within the tumor. In addition, our study cannot exclude other possibilities that those intratumoral novel tumor reactive $\mathrm{T}$ cell clones came from neither intratumoral sites nor draining LNs. For example, the tumor antigens can be released into peripheral blood, where antigen presenting cells can process and present tumor antigens to $\mathrm{T}$ cell and prime them, then the tumor reactive $\mathrm{T}$ cells retain and reside in primary tumors via peripheral blood circulation.

\section{Conclusion}

By integrative analysis of single-cell gene expression and immune profiling, our study revealed that all expanded clones identified in the sentinel $\mathrm{LN}$ were also present in the primary breast tumors while $10.91 \%$ of the expanded clones in the primary tumors were not found in the sentinel LNs, and these novel intratumoral $\mathrm{T}$ cell clones were characterized by high tissues retention capacity $($ CXCR6 +ITGAE +$)$ and a distinct coinhibitory pattern $(\mathrm{CD} 39+\mathrm{NKG2A}+)$ compared with the expanded T cell clones common to both sites. Multiplex immunofluorescence imaging showed the TLSs may play a role in eliciting non-sentinel LN-derived T cell clones. These results point to the need for exploring the role of TLS in antitumor immunity.

\section{Author affiliations}

${ }^{1}$ Department of Hepatobiliary Surgery, Nanjing University Medical School Affiliated Nanjing Drum Tower Hospital, Nanjing, Jiangsu, China

${ }^{2}$ Department of Pathology, Harbin Medical University, Harbin, Heilongjiang, China ${ }^{3}$ Department of Breast Surgery, Harbin Medical University Cancer Hospital, Harbin, Heilongjiang, China 
${ }^{4}$ Translational Medicine Research and Cooperation Center of Northern China, Heilongjiang Academy of Medical Sciences, Harbin, Heilongjiang, China ${ }^{5}$ Sino-Russian Medical Research Center, Harbin Medical University, Harbin, People's Republic of China

Acknowledgements We thank the Sugon Advanced Computing service platform and Suzhou Supercomputing Center for providing the HPC environment.

Contributors Conceived project: $\mathrm{SJ}$; performed experiments and collected data: all authors; analyzed data: SJ and QX; acquired funding: SJ, DPa nd TL; wrote manuscript: SJ and QX; guarantor: SJ.

Funding This work was supported by grants from Key Research Program of the Science and Technology Department of Jiangsu Province (BE2021602 to SJ), The Key Project of the Ministry of Science and Technology of China (SQ2021YFA090185 to SJ), The Key Project by Medical Science and Technology Development Foundation, Nanjing Department of Health (ZKX2020001 to SJ), National Natural Science Foundation of China (82072903 to TL), National Natural Science Foundation of China (81972706 to TL), Heilongjiang Province Natural Science Foundation Joint Guidance Project (LH2020H125 to TL), and Heilongjiang Province Returned Personnel Funding (21032190029 to TL).

Competing interests None declared.

Patient consent for publication Not applicable.

Ethics approval The study was conducted in accordance with the Declaration of Helsinki. Ethical approval was obtained from the Research Ethics Committee of Harbin Medical University Cancer Hospital.

Provenance and peer review Not commissioned; externally peer reviewed.

Data availability statement Data are available in a public, open access repository. All raw scRNA-seq and scTCR-seq will be deposited to GEO shortly, and the accession number will be provided when available.

Supplemental material This content has been supplied by the author(s). It has not been vetted by BMJ Publishing Group Limited (BMJ) and may not have been peer-reviewed. Any opinions or recommendations discussed are solely those of the author(s) and are not endorsed by BMJ. BMJ disclaims all liability and responsibility arising from any reliance placed on the content. Where the content includes any translated material, BMJ does not warrant the accuracy and reliability of the translations (including but not limited to local regulations, clinical guidelines, terminology, drug names and drug dosages), and is not responsible for any error and/or omissions arising from translation and adaptation or otherwise.

Open access This is an open access article distributed in accordance with the Creative Commons Attribution Non Commercial (CC BY-NC 4.0) license, which permits others to distribute, remix, adapt, build upon this work non-commercially, and license their derivative works on different terms, provided the original work is properly cited, appropriate credit is given, any changes made indicated, and the use is non-commercial. See http://creativecommons.org/licenses/by-nc/4.0/.

ORCID iD

Shiping Jiao http://orcid.org/0000-0001-6546-9369

\section{REFERENCES}

1 Long B, Brém E, Koyfman A. Oncologic emergencies: immunebased cancer therapies and complications. West J Emerg Med 2020;21:566-80.

2 Mellman I, Coukos G, Dranoff G. Cancer immunotherapy comes of age. Nature 2011;480:480-9.

3 Sautès-Fridman C, Petitprez F, Calderaro J, et al. Tertiary lymphoid structures in the era of cancer immunotherapy. Nat Rev Cancer 2019;19:307-25.

4 Hollern DP, Xu N, Thennavan A, et al. B cells and T follicular helper cells mediate response to checkpoint inhibitors in high mutation burden mouse models of breast cancer. Cell 2019;179:e1121:1191-206.
5 Cabrita R, Lauss M, Sanna A, et al. Tertiary lymphoid structures improve immunotherapy and survival in melanoma. Nature 2020;577:561-5.

6 Petitprez F, de Reyniès A, Keung EZ, et al. B cells are associated with survival and immunotherapy response in sarcoma. Nature 2020;577:556-60.

7 Jiao S, Subudhi SK, Aparicio A, et al. Differences in tumor microenvironment dictate $\mathrm{T}$ helper lineage polarization and response to immune checkpoint therapy. Cell 2019;179:e1113:1177-90.

8 Xiong Q, Peng C, Yan X, et al. Characteristics of SARS-CoV-2specific cytotoxic $T$ cells revealed by single-cell immune profiling of longitudinal COVID-19 blood samples. Signal Transduct Target Ther 2020;5:285

9 Yost KE, Satpathy AT, Wells DK, et al. Clonal replacement of tumorspecific T cells following PD-1 blockade. Nat Med 2019;25:1251-9.

10 Waks AG, Winer EP. Breast cancer treatment: a review. JAMA 2019;321:288-300.

11 Stuart T, Butler A, Hoffman P, et al. Comprehensive integration of single-cell data. Cell 2019;177:1888-902.

12 Yost KE, Satpathy AT, Wells DK, et al. Clonal replacement of tumorspecific T cells following PD-1 blockade. Nat Med 2019;25:1251-9.

13 Bergen V, Lange M, Peidli S, et al. Generalizing RNA velocity to transient cell states through dynamical modeling. Nat Biotechnol 2020;38:1408-14.

14 La Manno G, Soldatov R, Zeisel A, et al. RNA velocity of single cells. Nature 2018;560:494-8.

15 Makki J. Diversity of breast carcinoma: histological subtypes and clinical relevance. Clin Med Insights Pathol 2015;8:23-31.

16 Patel AP, Tirosh I, Trombetta JJ, et al. Single-cell RNA-seq highlights intratumoral heterogeneity in primary glioblastoma. Science 2014;344:1396-401.

17 Puram SV, Tirosh I, Parikh AS, et al. Single-cell transcriptomic analysis of primary and metastatic tumor ecosystems in head and neck cancer. Cell 2017;171:1611-24.

18 Tirosh I, Izar B, Prakadan SM, et al. Dissecting the multicellular ecosystem of metastatic melanoma by single-cell RNA-seq. Science 2016;352:189-96.

19 Tian T, Li X, Hua Z, et al. S100A7 promotes the migration, invasion and metastasis of human cervical cancer cells through epithelialmesenchymal transition. Oncotarget 2017;8:24964-77.

20 Srikrishna G. S100A8 and S100A9: new insights into their roles in malignancy. J Innate Immun 2012;4:31-40.

21 Zhang Y, Zheng L, Zhang L, et al. Deep single-cell RNA sequencing data of individual $T$ cells from treatment-naïve colorectal cancer patients. Sci Data 2019;6:131

22 Li X-Y, Moesta AK, Xiao C, et al. Targeting CD39 in cancer reveals an extracellular ATP- and Inflammasome-Driven tumor immunity. Cancer Discov 2019:9:1754-73.

23 van Montfoort N, Borst L, Korrer MJ, et al. NKG2A blockade potentiates CD8 T cell immunity induced by cancer vaccines. Cell 2018:175:1744-55

24 Chambers CA, Kuhns MS, Egen JG, et al. CTLA-4-mediated inhibition in regulation of $T$ cell responses: mechanisms and manipulation in tumor immunotherapy. Annu Rev Immunol 2001;19:565-94.

25 Colpitts SL, Dalton NM, Scott P. II-7 receptor expression provides the potential for long-term survival of both CD62Lhigh central memory T cells and Th1 effector cells during Leishmania major infection. $J$ Immunol 2009;182:5702-11.

26 van der Leun AM, Thommen DS, Schumacher TN. CD8 ${ }^{+} \mathrm{T}$ cell states in human cancer: insights from single-cell analysis. Nat Rev Cancer 2020;20:218-32.

27 Schirrmacher V, Feuerer M, Fournier P, et al. T-cell priming in bone marrow: the potential for long-lasting protective anti-tumor immunity. Trends Mol Med 2003;9:526-34.

28 Helmink BA, Reddy SM, Gao J, et al. B cells and tertiary lymphoid structures promote immunotherapy response. Nature 2020;577:549-55.

29 Mellman I, Steinman RM. Dendritic cells: specialized and regulated antigen processing machines. Cell 2001;106:255-8.

30 Trombetta ES, Mellman I. Cell biology of antigen processing in vitro and in vivo. Annu Rev Immunol 2005;23:975-1028. 